\title{
NANOPHOTONICS
}

\section{Light-emitting indium tin oxide}

Indium tin oxide (ITO) is an important conducting oxide material that is widely used as a transparent electrode in solar cells and flat-panel displays. Usually this material is fabricated as a thin planar film, but in recent years there has been considerable interest in fabricating ITO in the form of nanostructures, especially nanowires, for use in miniature photonic circuitry.

The transparency and conductivity of an ITO nanowire strongly depend on its tin concentration. Unfortunately, however, controlling tin concentration in ITO nanowires is difficult through existing growth techniques. Now, Jing Gao and co-workers from Singapore and China have developed a method of fabricating ITO nanowires that not only provides control over the tin concentration but also introduces light-emitting functionality, which could prove useful for optoelectronic devices (Nanotechnology 22, 195706; 2011).

The single-crystalline ITO nanowires were synthesized through a vapour transport method, in which $\ln _{2} \mathrm{O}_{3}, \mathrm{SnO}_{2}$ and graphite powders were loaded into a horizontal tube furnace. The nanowires were grown at $840^{\circ} \mathrm{C}$ in an argon gas atmosphere on a quartz substrate coated with thin sputtered layers of gold, which acted as a catalyst. The fastest nanowire growth was in the

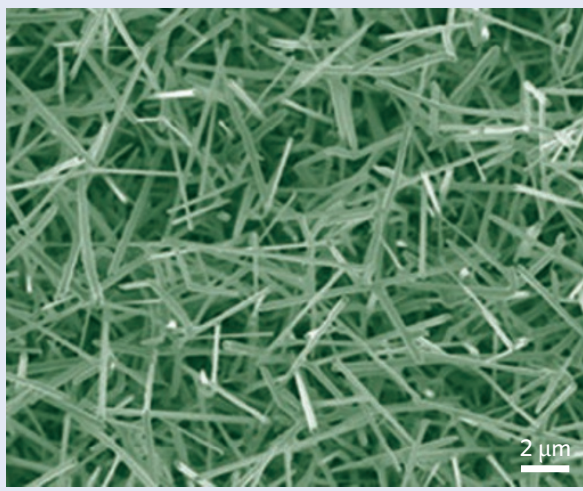

[100] direction. The resulting nanowires were approximately $180 \mathrm{~nm}$ wide and a few micrometres in length. The atomic doping of tin in the ITO nanowires was tuned in the range of $0-6.4 \%$ (atomic, at. \%) by controlling the composition of the source materials.

The nanowires were transparent in the visible and near-infrared regions of the electromagnetic spectrum. Their absorption edge shifted from $3.4 \mathrm{eV}$ to $3.8 \mathrm{eV}$ when the tin doping was increased from 3.8 at.\% to 6.4 at.\%. When the nanowires were excited by a $\mathrm{He}-\mathrm{Cd}$ laser (wavelength of $325 \mathrm{~nm}$ ), broad $430 \mathrm{~nm}$. The researchers believe that the light emission was due to the formation of a new defect level induced in the blue emission was observed at around

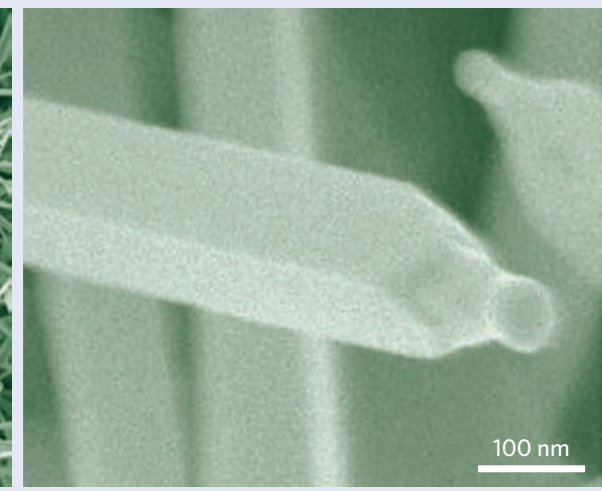

bandgap by the tin doping. A strong and sharp emission peak was observed at $380 \mathrm{~nm}$ for ITO nanowires with a tin doping of 3.8 at.\%. The temperature dependence of the photoluminescence revealed the existence of bound exciton complexes around $3.36 \mathrm{eV}$ below $100 \mathrm{~K}$. The researchers also demonstrated the epitaxial growth of vertically aligned ITO nanowires on an $\alpha$-cut sapphire substrate. $X$-ray diffraction measurements showed that the epitaxial growth direction of ITO [001] was aligned with the [110]oriented sapphire substrate. These findings are promising for the future integration of blue-emitting ITO into optoelectronic devices.
NORIAKI HORIUCHI

\section{ULTRAFAST OPTICS}

\section{Focusing through scattering media}

A scattering medium such as biological tissue distorts the propagation of light pulses in both space and time, making tasks such as focusing and imaging problematic. Fortunately, careful manipulation of the light field's spatial phase prior to entering the medium can help mitigate such distortions and open new prospects for nonlinear microscopy.

\section{Andrew M. Weiner}

T he scattering of waves propagating through inhomogeneous media is of interest across diverse areas of physics and engineering, including acoustics, radiofrequency wireless communication, optics and electron transport. In the optical domain, scattering-induced wavefront distortions limit focusing ability and degrade image quality. Adaptive optics can be used to address weak phase aberrations, such as those encountered in free-space optical communications and astronomical imaging through the atmosphere. However, compensating for the distortions that occur in strongly scattering media such as biological tissue is a more significant challenge.
In the case of diffusive light propagation, the output waveform is a noise-like (speckle) field, which generally shows no discernible resemblance to the input wavefront. However, by programming the spatial phase of the input field using a modern spatial light modulator, coherent light can be 'focused' through an opaque 